\title{
New polymeric sorbent for the solid-phase extraction of indole-3-acetic acid from plants followed by liquid chromatography - Fluorescence detector
}

\author{
Beatrice Campanella $^{\mathrm{a}, \mathrm{b}}$, Elena Pulidori ${ }^{\mathrm{a}}$, Massimo Onor ${ }^{\mathrm{b}}$, Elisa Passaglia ${ }^{\mathrm{b}}$, Stefania Tegli ${ }^{\mathrm{c}}$, \\ Carlos García Izquierdo ${ }^{\mathrm{d}}$, Emilia Bramanti ${ }^{\mathrm{b}, *}$ \\ a Università di Pisa, Dipartimento di Chimica e Chimica Industriale, via Moruzzi 13, 56124 Pisa, Italy \\ b Consiglio Nazionale delle Ricerche, Istituto di Chimica dei Composti Organometallici, UOS di Pisa, via Moruzzi 1, 56124 Pisa, Italy

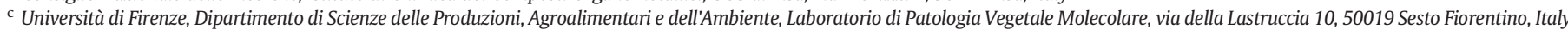 \\ d Consejo Superior de Investigaciones Cientificas CEBAS-CSIC, Murcia, Spain
}

\section{A R T I C L E I N F O}

\section{Article history:}

Received 2 March 2016

Received in revised form 14 April 2016

Accepted 14 April 2016

Available online 22 April 2016

\section{Keywords:}

Indole-3-acetic acid

Molecular fluorescence

Plants

Polymeric sorbent

\begin{abstract}
A B S T R A C T
Plant hormones play a crucial role in controlling plant growth and development. These groups of naturally occurring substances trigger physiological processes at very low concentrations, which require sensitive techniques for their quantitation.

This study reports on the development of a newly synthesized polymer sorbent for the analysis of indole-3-acetic acid (IAA), which is the most important auxin in plants, from complex raw matrices, such as plant extracts. IAA was determined by high-performance liquid chromatography (HPLC) coupled with fluorescence detection (FD). The polymer developed was able to bind about $94 \%$ of IAA with a reproducible manner. The release of IAA in methanol was more than $70 \%$ for both of them. Non-specific hydrophobic interactions are, likely, the dominant driving force of the binding between IAA and the polymer.

This methodology was applied for the determination of IAA in raw lemon leaves. The detection and quantification limits for IAA in real samples were $0.50 \pm 0.08 \mathrm{ng} / \mathrm{g}$ and $2.64 \pm 0.09 \mathrm{ng} / \mathrm{g}(\mathrm{n}=3, \alpha=0.05)$, respectively. As expected, the IAA concentration in uninfected lemon sample was significantly lower respect to the concentration found in infected sample.
\end{abstract}

(c) 2016 Elsevier B.V. All rights reserved.

\section{Introduction}

Plant hormones, a group of naturally occurring substances, are mediators of endogenous processes to regulate plant growth, development and the ability to properly respond to biotic and abiotic stresses [1-3]. The main classes of phytohormones are auxins, abscisic acid, cytokinins, ethylene and gibberellins. In general, they differentially contribute to numerous important plant biological processes, such as cell division, enlargement and differentiation, organ formation, seed dormancy and germination, organ senescence and abscission [4-6]. As phytohormones are synthesized in plants at trace and ultra-trace levels $(0.1-50 \mathrm{ng} / \mathrm{g}$ fresh weight) [7], it is necessary to develop simple and effective methods to isolate, purify and detect them in plant tissues. Moreover, as the optimum endogenous level of each phytohormone must be tightly controlled, these molecules are mainly present in their conjugated forms, which are not active. Thus, from a biological point of view, only the free form present in low percentages is of interest [8]. Moreover, the fine-tuned balance of the different phytohormones and their

\footnotetext{
* Corresponding author.

E-mail address: bramanti@pi.iccom.cnr.it (E. Bramanti).
}

conjugated forms is essential for plants to increase their resistance to phytopathogens. As far as the plant pathogenic bacteria are concerned, most of them are also able to synthesize and conjugate IAA, with the aim to hijack the host auxin metabolism to increase plant susceptibility [3].

Together with molecular biology approaches, several analytical methods have been developed for the determination of phytohormones in plants, mainly based on high performance liquid chromatography (HPLC) coupled with mass spectrometry (MS or MS/MS) [9]. In MS analysis the use of isotope labeled compounds (expensive and not always available) is mandatory to compensate for ionization suppression caused by co-eluting components in the plant matrix [10].

Due to the low concentration of phytohormones in plant tissue and the presence of interfering substance, sample pretreatment represents the most critical and time-consuming step, requiring extensive purification prior to final quantitation. Among the recently developed pretreatment methods, dispersive liquid-liquid microextraction (DLLME) [11] and solid-phase microextraction (SPME) [12] are characterized by high enrichment ability and low organic solvent consumption, but they suffer from low recovery and reproducibility. Hollow fiber-based liquid-liquid microextraction [13] requires little organic solvent, and 
offers high enrichment factor, but it is limited to the extraction of ionisable and chargeable compounds from aqueous samples. Traditional liquid-liquid extraction, one of the simplest methods for sample pretreatment, suffers from the loss of compound of interest. Solid-phase extraction (SPE) is recognized as the most common pre-treatment technique for sample clean-up and preconcentration. The commercial sorbents are usually silica and bonded silica such as C8 and C18, which suffer from the non-specific selectivity in isolating plant hormones from plants (e.g. C18 cartridges are capable of retaining lipids and plant pigments in the crude extract) [9].

In the last ten years molecularly imprinted solid-phase extraction, which combines the advantages of both molecular recognition and traditional SPE methods, has attracted more interest [14]. In an effort to develop selective sorbents, which will speed up isolation of auxins from plant extracts, several molecular imprinted polymers (MIPs) specific for IAA were synthesized and characterized [15-17]. The synthesis of MIPs based on non-covalent interaction (hydrogen bonding, and hydrophobic interactions) has been widely used due to its simplicity and flexibility. However, the interaction between monomers and template molecule is relatively weak, especially for small template molecule, which gives low sample load capacity. Another limitation of MIPs is that they are synthesized in the presence of large quantities of template. Thus, despite their extensive washing, which is preliminary to their use, traces of template can be released during sample pretreatment, which may interfere with the analysis. MIP use may be also limited by their low yield of specific binding sites [14]. In particular, non-specific interactions are common with MIPs synthesized in a non-covalent imprinted mode [18].

In this work we synthesized a new polymer for the pre-treatment of plant extracts for the identification and quantitation of IAA, which is the most important auxin in plants, from complex raw matrices, such as plant extracts. The idea was to use a typical synthesis procedure commonly employed for MIPs in the absence of the template. The synthetized polymer is constituted by vinylpyridine cross-linked with trimethylolpropane trimethacrylate. This polymer is quite different from the traditional, commercially available SPE sorbent for reversed phase pre-treatment, which are based on alkyl-bonded silica or styrene-divinylbenzene.

In this work, we determined IAA in raw lemon leaves. After its pre-treatment, IAA was separated and quantified by high-performance liquid chromatography (HPLC) with fluorescence detection (FD). Molecular fluorescence is an excellent alternative to high-cost mass spectrometric techniques, due to its sensitivity and selectivity.

\section{Experimental}

\subsection{Chemicals and reagents}

Indole-3-acetic acid (IAA), salicylic acid (SA), abscisic acid (ABA), 1naphthalenacetic acid (NAA), catechin, and indole 3-butyrric acid (IBA) were purchased from Sigma-Aldrich (Milan, Italy). Stock solutions of IAA, SA, ABA, NAA, catechin and IBA were prepared in methanol (Chromasolv for HPLC $\geq 99.9 \%$ ). Each solution preparation was accomplished using adjustable pipettes and, for better precision, all aliquots were weighted. Working solutions were prepared from stock solution by dilution in $10 \%$ or $50 \%$ methanol.

Ultra-pure water was obtained using a Milli-Q system (Purerlab Pro + Purelab Classic, Millipore, USA), and it was used in all experiments.

For the synthesis, 4-vinylpyridine (4-VP, 95\%; contains $100 \mu \mathrm{g} / \mathrm{mL}$ hydroquinone as inhibitor), 2,2'-azobis(2-methylpropionitrile) (AIBN, 98\%) and trimethylolpropanetrimethacrylate (TRIM, 98\%) were purchased from Sigma-Aldrich (Milan, Italy). Acetonitrile (LC-MS Chromasolv $>99.9 \%$ ) and formic acid (for mass spectrometry, 98\%) were purchased from Sigma-Aldrich-Fluka. C18 Sep-Pak (30 mg, C18 stationary phase) and HLB Oasis (30 mg, N-vinylpyrrolidone and divinylbenzene stationary phase) cartridges were purchased from Water Corporation.

\subsection{Instrumentation and chromatographic conditions}

An HPLC gradient pump (P4000, ThermoFinnigan) was coupled with a vacuum membrane degasser (SCM1000, ThermoFinnigan), an AS3000 autosampler (ThermoFinnigan), a UV6000 diode array detector and a FL3000 fluorescence detector (ThermoFinnigan).

Separations of phytohormones were carried out using a reversedphase HPLC column ZORBAX Eclipse Plus C18 (4.6 mm × $100.0 \mathrm{~mm}$, $3.5 \mu \mathrm{m}$, Agilent Technologies), equipped with a guard column ( $4.6 \mathrm{~mm} \times 12.5 \mathrm{~mm}, 5.0 \mu \mathrm{m}$, Agilent). Column temperature was set at $40{ }^{\circ} \mathrm{C}$ and injection volume was $50 \mu \mathrm{L}$.

The determination of IAA in standard solutions was performed using an isocratic elution in $40 \%$ methanol-60\% water ( $0.1 \%$ formic acid). Mobile phases for the determination of IAA in plant samples consisted of $40 \%$ methanol- $0.1 \%$ formic acid in water (eluent $A$ ) and $0.1 \%$ formic acid in methanol (eluent $B$ ). The gradient was as follows: $0-15$ min, $100 \% \mathrm{~A} ; 15-20$ min, linear gradient up to $100 \% \mathrm{~B} ; 20-35$ min $100 \% \mathrm{~B}$; 35-37 min, linear gradient up to $100 \%$ A. Post-run time was 15 min. Elution was performed at a solvent flow rate of $0.8 \mathrm{~mL} \mathrm{~min}^{-1}$.

The detection of $A B A$ and catechin was performed in absorbance at $273 \mathrm{~nm}$; the detection of SA was performed in absorbance at $243 \mathrm{~nm}$; the detection of IAA, IBA and NAA was performed in fluorescence using $\lambda_{\mathrm{ex}}=280 \mathrm{~nm}$ and $\lambda_{\mathrm{em}}=340 \mathrm{~nm}$.

ChromQuest $^{\mathrm{TM}} 4.2$ Chromatography Data System was used to carry out HPLC-DAD/FD control, data acquisition and data analysis. Excel 2007 by Microsoft Corporation was used for the statistical treatment of the chromatographic data.

\subsection{Polymer synthetic procedure}

The following procedure has been selected on the basis of the synthesis of MIPs reported in literature [19].

In a Schlenk tube one hundred ninety microliters of 4-VP (1.77 mmol, as functional monomer), $3.2 \mathrm{~mL}$ of TRIM ( $8.86 \mathrm{mmol}$, as crosslinker) and $0.041 \mathrm{~g}$ AIBN ( $0.25 \mathrm{mmol}$, as radical initiator) were dissolved in $10 \mathrm{~mL}$ methanol previously degassed, under nitrogen atmosphere. The mixture was purged with nitrogen gas to remove the oxygen that could inhibit the polymerization. The glass tube was sealed and the thermal polymerization was performed by heating at $60{ }^{\circ} \mathrm{C}$ for $20 \mathrm{~h}$. A yellow powder was obtained (the resulting polymer swollen by solvent) that was ground in a mortar and dried under vacuum, till constant weight. Subsequently, the polymer was washed 6 times with methanol/acetic acid $(9 / 1 \mathrm{v} / \mathrm{v})$ in order to remove the unreacted monomers and the by-products from the synthesis. The final yield was $98.6 \%$.

This procedure was performed twice, obtaining two batches of the polymer in order to test its reproducibility.

\subsection{Bacterial strains, growth conditions, and plant inoculation trials}

The strain BAC3 of Pseudomonas syringae pv. syringae (Pss), virulent on lemon plants, was used in this study to carry out inoculation trials. Bacteria were grown overnight in vitro on King's B medium (KB) [20], amended with Nitrofurantoine $(50 \mathrm{mg} / \mathrm{L}$ ) at CEBAS (Murcia, Spain). Then, the bacterial suspensions were washed twice in sterile physiological solution (SSF, $0.85 \mathrm{~g} \mathrm{NaCl} / 100 \mathrm{~mL} \mathrm{H}_{2} \mathrm{O}$ ), and their concentration adjusted to $10^{9} \mathrm{UFC} / \mathrm{mL}$.

Ten lemon (Citrus $\times$ limon) plants growth in field at CEBAS were inoculated with $10 \mu \mathrm{L}$ of a concentrated Pss suspension $\left(10^{9} \mathrm{UFC} / \mathrm{mL}\right)$. After two months three leaves from each plant were selected, washed with ultrapure water and dried. Then, 10 leaves were selected randomly, freezed at $-20{ }^{\circ} \mathrm{C}$ for $24 \mathrm{~h}$, lyophilized and stored at $-20{ }^{\circ} \mathrm{C}$ until used for analytical investigations. 


\subsection{Sample preparation}

We report here the final analytical procedure developed in this work and applied for the pre-treatment of plant extracts for the identification and quantitation of IAA. One milliliter $80 \%$ methanol was added to $25 \mathrm{mg}$ aliquots of the lyophilized lemon leave samples. A series of spiked samples with IAA was also analyzed for the internal calibration.

The extraction was made by magnetic stirring at $0{ }^{\circ} \mathrm{C}$ for $30 \mathrm{~min}$. After centrifugation ( $8600 \mathrm{~g}, 4^{\circ} \mathrm{C}$ for $30 \mathrm{~min}$ ), the supernatant was transferred to a new vial and the methanol was partially removed under nitrogen flow. A suitable amount of water was added to reach approximately $10 \%$ methanol. This solution was added to $30 \mathrm{mg}$ of the polymer previously conditioned. The resulting suspension was vortexed for $5 \mathrm{~min}$ at room temperature, centrifuged $\left(8600 \mathrm{~g}, 20^{\circ} \mathrm{C}\right.$ for $\left.10 \mathrm{~min}\right)$ and the supernatant was wasted. The loaded polymer was further washed by adding $1 \mathrm{~mL} \mathrm{10 \%} \mathrm{methanol} \mathrm{in} \mathrm{order} \mathrm{to} \mathrm{remove} \mathrm{eventual}$ interfering compounds. The resulting suspension was vortex for $5 \mathrm{~min}$ at room temperature, centrifuged $\left(8600 \mathrm{~g}, 20^{\circ} \mathrm{C}\right.$ for $\left.10 \mathrm{~min}\right)$ and the supernatant was wasted. Thus, the polymer was treated with $1 \mathrm{~mL}$ of $100 \%$ methanol for the release of IAA and other eventual extracted compounds. The resulting suspension was vortexed for $5 \mathrm{~min}$ at room temperature and centrifuged $\left(8600 \mathrm{~g}, 20^{\circ} \mathrm{C}\right.$ for $\left.10 \mathrm{~min}\right)$. The supernatant was filtered using a Uniprep syringeless filtration device $(0.25 \mu \mathrm{m}$, Agilent Technologies) and the methanol was removed by $50 \%$ under nitrogen flow. A suitable amount of water was added to restore the initial volume. The resulting solutions were analyzed by HPLC-DAD/FD.

\section{Results and discussion}

The aim of our work was the development of an easy and straightforward strategy for the analysis of 3-IAA. This molecule is indeed of great interest for phytopathologist, as it is strongly correlated with the health status of the plant. Thus, it is advisable a method for the quantification of 3-IAA in plant sample which is fast and simple.

The new synthetized polymer was first tested for the binding and release of IAA standard solutions. For the binding test, 10\% methanol-90\% water was selected as solvent. IAA has, indeed, a poor solubility in water $\left(1.5 \mathrm{~g} / \mathrm{L}\right.$ at $\left.20{ }^{\circ} \mathrm{C}\right)$ [21]. Thus, this solvent composition should, in principle, favor the interaction of IAA with the polymer. The polymer was always conditioned before its use with the same solvent employed for the binding test. Methanol was selected as releasing solvent because of the high solubility of IAA in methanol.

The determination of IAA in the solution after the treatment with the polymer was performed by HPLC-DAD/FD using calibration curves in $50 \%$ methanol-50\% water $(5.00-40.00 \mathrm{ng} / \mathrm{mL}$ tested linear range; slope $\left.3.04 \pm 0.02 \cdot 10^{4} ; R^{2}=1.00\right)$.

The amount of IAA in the solution analyzed was calculated on the basis of the HPLC peak area (IAA $t_{R}=6.35 \mathrm{~min}$ ) and of the calibration curve. The percentage of IAA bound to the polymer was calculated with respect to the IAA analytical concentration employed for the test (20 ng/mL).

Table 1 summarizes the percentage values relative to the amount of IAA bound and IAA released, calculated with respect to the amount of IAA bound.

The polymer tied a significantly high percentage of IAA (about 95\%) and about $70 \%$ of IAA bound was released by methanol. No significant differences were observed between the two batches, showing that the polymer characteristics are reproducible.

\section{Table 1}

Percentage values relative to the amount of IAA bound to the polymer and the amount released. The values are expressed as average value \pm confidence interval $(n=3, \alpha=0.05)$.

\begin{tabular}{lll}
\hline Polymer & IAA bound & IAA released \\
\hline Batch 1 & $95 \pm 4$ & $70 \pm 3$ \\
Batch 2 & $93 \pm 3$ & $72 \pm 2$ \\
\hline
\end{tabular}

It is likely to hypothesize that the interaction of IAA with polymers is due to hydrophobic interactions and, in particular, to the $\pi-\pi$ stacking between IAA and the functional monomer (4-VP). In order to show that in this case the hydrophobic interactions are the dominant driving force of the binding, considering that acetonitrile does not favor the $\pi-\pi$ stacking [22], we performed a binding test in acetonitrile, following the same procedure described above, with the release in methanol. We found that for both polymers only $10 \%$ IAA was retained. Thus, we definitely concluded that the interactions between IAA and the polymer are due to the hydrophobic/ $\pi-\pi$ interactions.

Fig. 1 shows representative fluorescence chromatograms of the solution after the binding test of $20 \mathrm{ng} / \mathrm{mL}$ standard solution of IAA in $10 \%$ methanol on the polymer batch 2 (as example), compared with the chromatogram of the $20 \mathrm{ng} / \mathrm{mL}$ IAA standard solution (as reference) and the methanol solution used for IAA release.

The selectivity of the synthesized polymer with respect to other common phytohormones was evaluated treating $30 \mathrm{mg}$ of conditioned polymer with $1 \mathrm{~mL}$ of standard solutions containing $1 \mu \mathrm{g} / \mathrm{mL}$ of $\mathrm{SA}, \mathrm{ABA}$, catechin (detected by DAD) and $20 \mathrm{ng} / \mathrm{mL}$ of IAA, IBA, NAA (detected by FD) dissolved in $10 \%$ methanol-90\%water using the same procedure previously described. Table 2 shows the percentage values relative to the amount of analytes bound and released.

The results of Table 2 show that the polymer is able to bind also the other phytohormones investigated with binding percentage $>70 \%$. We can hypothesize that phytohormones, as well as IAA, are bound through hydrophobic interactions with the polymeric matrix. ABA is less retained (48\%) likely because it does not have an aromatic structure and, therefore, it cannot bind to the polymer through $\pi-\pi$ stacking interactions. Also for the phytohormones tested the release in methanol was high (>70\%).

On the basis of these results we can conclude that he polymer is suitable for the pre-treatment of plant extracts for the identification and quantitation of IAA and also for the identification of the other tested phytohormones (SA, ABA, catechin, IBA, NAA).

In the following section we present the application of the polymer for the pre-treatment of plant extracts for the identification and determination of IAA and eventual other phytohormones in real samples. The application of commercial SPE HLB and C18 cartridges was also performed in real samples as comparison.

\subsection{Application of the polymer to the pre-treatment of IAA in plant extracts}

Extracts from lemon leaves infected or not with Pseudomonas were employed for the determination of IAA using the polymer.

These samples have been provided by CEBAS (Murcia, Spain) in the framework of the Life + ENV/IT/336-After Cu project, which aims to replace copper treatments in plants with anti-infective environmental friendly molecules against plant pathogenic bacteria.

It is known that IAA is an essential phytohormone, with profound effects on plant growth and development as well as stress responses. Also the production of IAA by plant-associated bacteria is an important aspect of research on IAA metabolism, although the role of IAA production by bacteria has not completely unveiled yet [23].

Fig. 2A shows a representative fluorescence chromatogram at $340 \mathrm{~nm}$ of the extracts purified on the polymer following the procedure described in the experimental section. The extracts were from bacteriainfected lemon leaves not spiked (black line) and spiked (red line) with $20 \mathrm{ng} / \mathrm{mL}$ IAA.

Fig. 2A shows the increase of the peak at $5.84 \mathrm{~min}$ of IAA in the spiked sample. No other peaks increased in the chromatogram. Despite the complexity of the matrix, this result shows that the treatment of the sample extract with the polymer allowed the identification and quantitation of IAA. As comparison, Fig. 2B shows the chromatogram of the same sample extracted three times with ethyl acetate, evaporated to dryness and redissolved in methanol. However, with this treatment was not possible to separate 3-IAA from the interfering peaks. 


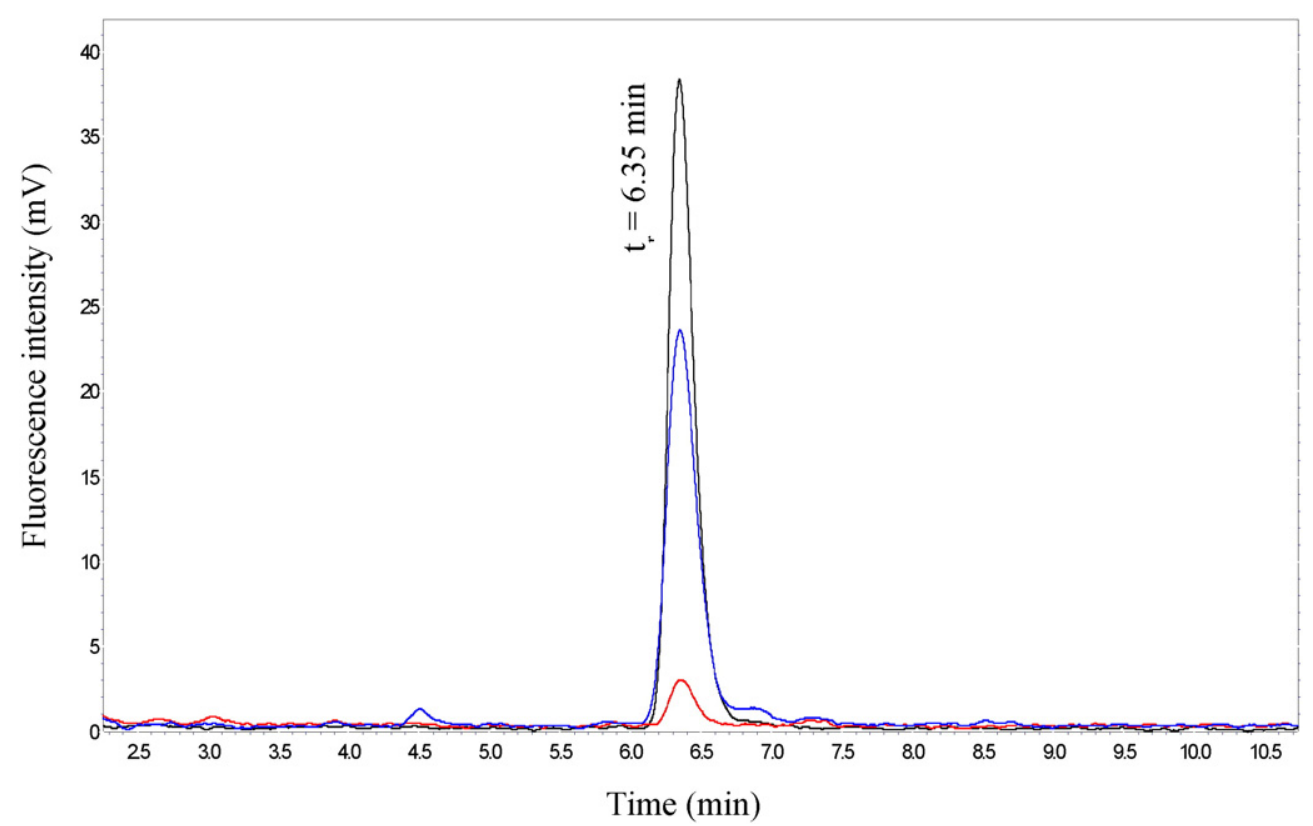

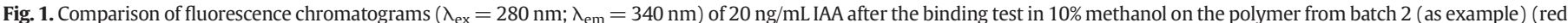
curve), compared with the chromatogram of untreated $20 \mathrm{ng} / \mathrm{mL}$ IAA (as reference, black curve) and the methanol solution used for IAA release from the polymer (blue curve).

The external calibration curve on the polymer was performed by loading standard solutions at different concentrations of IAA prepared in $10 \%$ methanol to different aliquots of the conditioned polymer (30 mg). The internal calibration curve was performed spiking $25 \mathrm{mg}$ aliquots of lyophilized plant samples with IAA standard solutions at different concentrations and following the procedure previously described (see paragraph 2.6).

Table 3 summarizes the fitting parameters of the external and internal calibration curves.

The matrix effect was evaluated by the ratio of the slopes of the internal and external calibration curves. The recovery was $49 \pm 5 \%$ for the bacterium infected samples and $58 \pm 5 \%$ for the not infected samples. These values were not significantly different.

The limit of detection and quantification, determined as the analyte concentration corresponding to the smallest signal that is distinguishable from the background noise plus three and ten times its standard deviation, were $0.50 \pm 0.08 \mathrm{ng} / \mathrm{g}$ and $2.64 \pm 0.09 \mathrm{ng} / \mathrm{g}(\mathrm{n}=3, \alpha=$ $0.05)$, respectively.

Using standard addition method, we found that the IAA concentration was $7.73 \pm 0.08 \mathrm{ng} / \mathrm{g}$ in uninfected lemon sample, while these values raised to $40.12 \pm 0.09 \mathrm{ng} / \mathrm{g}$ in Pss infected lemon sample $(\mathrm{n}=$ $3, \alpha=0.05$ ). The higher concentration of IAA found in bacterial infected leaves was coherent with the expected increase in plant metabolism for IAA due to the bacterial infection, and to the contribution of IAA produced by Pss [23].

Table 2

Percentage values relative to the amount of SA, ABA, catechin, IAA, IBA, NAA bound to the polymer and the percentage released in methanol. The values are expressed as average value \pm confidence interval ( $\mathrm{n}=3, \alpha=0.05)$.

\begin{tabular}{lcc}
\hline Analytes & Bound (\%) & Release (\%) \\
\hline Catechin & $74 \pm 3$ & $100 \pm 2$ \\
IAA & $87 \pm 2$ & $86 \pm 1$ \\
IBA & $100 \pm 2$ & $85 \pm 3$ \\
NAA & $93 \pm 1$ & $71 \pm 3$ \\
SA & $76 \pm 3$ & $67 \pm 2$ \\
ABA & $48 \pm 1$ & $85 \pm 3$ \\
\hline
\end{tabular}

Table 4 summarizes the most recent method developed for IAA quantitation in plant tissue by liquid chromatography coupled with UV or molecular fluorescence detection, compared with our method.

The LOD found using our method is comparable with that found in [12] on plant xylem fluids, a less complex matrix with respect to plant extracts.

3.2. Use of commercial C18 and HLB cartridges for the determination of IAA in plant extracts

The infected lemon plant extracts employed for the application of the polymer to IAA analysis were also treated, as comparison, with commercial C18 and HLB cartridges for the identification of IAA and its determination by HPLC-DAD/FD.

Fig. 3 shows representative fluorescence chromatograms at $340 \mathrm{~nm}$ $\left(\lambda_{\text {ex }}=280 \mathrm{~nm}\right)$ of lemon extracts treated with (A) C18 and (B) HLB cartridges, spiked or not with $10 \mathrm{ng} / \mathrm{mL}$ IAA.

The resulting chromatograms did not evidence significant difference among spiked or not spiked samples. Moreover, other compounds were extracted and coeluted with IAA, likely due to the non-specific adsorption of commercial polymeric sorbents. Moreover, we had to diluted these samples 10 times before their injection in the HPLC system in order to avoid the saturation of the fluorimetric detector.

Thus, our polymer was more effective than commercial C18 and HLB cartridges for the identification and quantitation of IAA in raw samples.

In perspective, the polymeric matrix developed in this work could be applied as a reversed phase with characteristics intermediate between those of silica with alkyl chains (e.g. C18) and those completely aromatic such as styrene/divinylbenzene (e.g. HLB).

\section{Conclusions}

In this work we synthesized a new polymeric sorbent for the pretreatment of plant extracts for the identification and quantitation of IAA, based on vinylpyridine cross-linked with trimethylolpropane trimethacrylate. In this specific case we exploit the aspecific interactions of the polymeric matrix of MIPs. It is well known, indeed, that aspecific binding interactions are very common in MIPs synthesized in a non- 

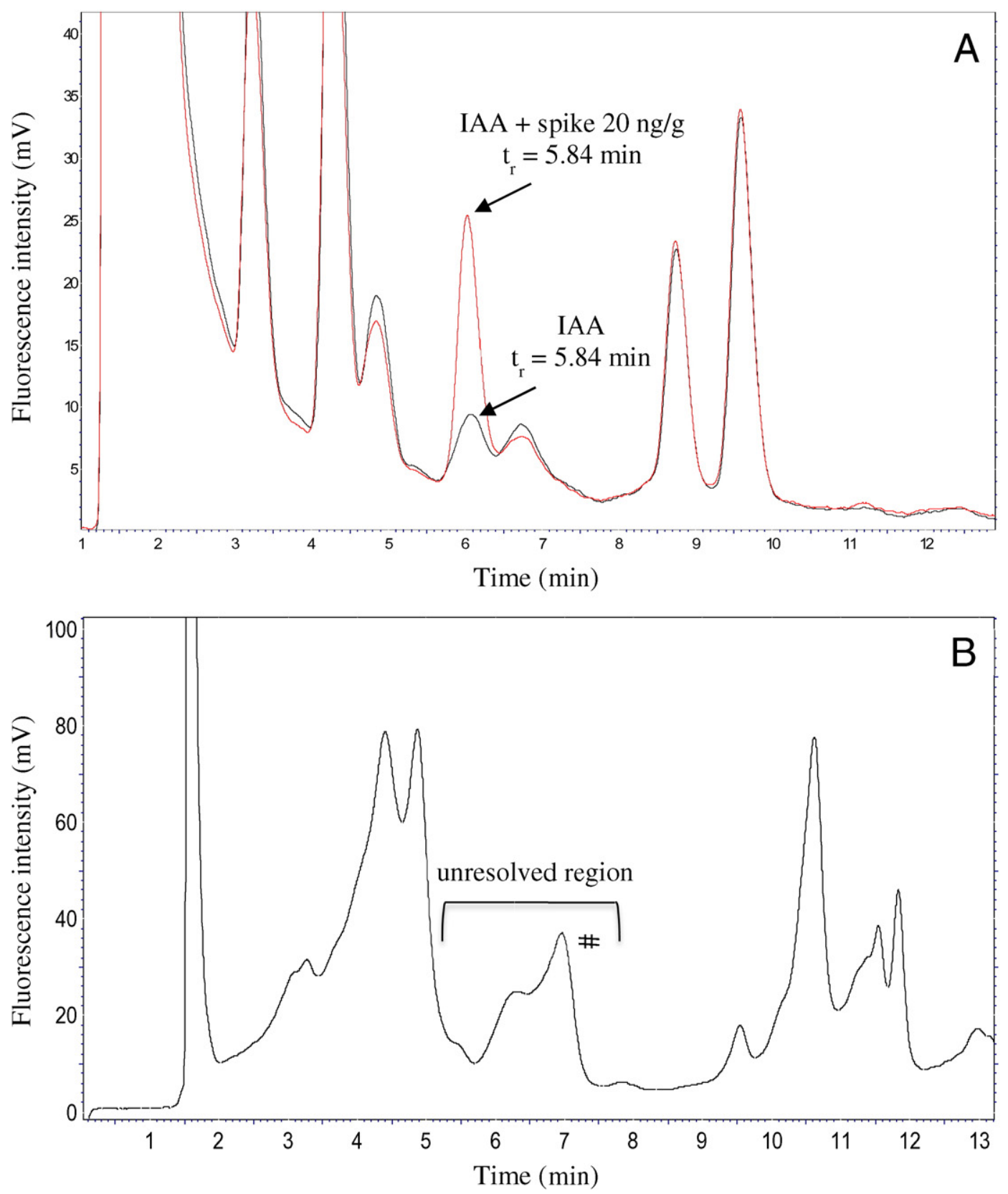

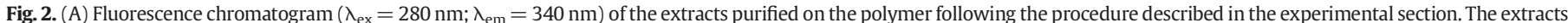

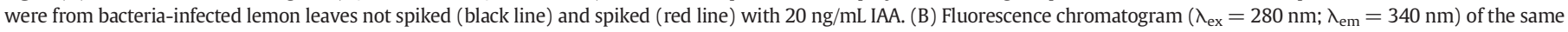
sample pre-treated by liquid-liquid extraction.

covalent imprinted mode [18]. For this reason we followed a typical non-covalent synthesis of MIPs.

The polymer developed was able to bind $95 \pm 4 \%$ (first batch) and $93 \pm 3 \%$ (second batch), demonstrating a reproducibility of its performance in the binding of IAA. The release of IAA in methanol was more than $70 \%$ for both of them. Non-specific hydrophobic interactions

Table 3

Fitting parameters of the external and internal calibration curves for the determination of IAA in extracts of lemon leave infected or not with bacteria using the polymer for its purification.

\begin{tabular}{llll}
\hline & $\begin{array}{l}\text { Range } \\
(\mathrm{ng} / \mathrm{g})\end{array}$ & Slope $\pm \mathrm{SD}$ & $\mathrm{R}^{2}$ \\
\hline Internal calibration (Pss infected samples) & $2.00-40.00$ & $8.6 \cdot 10^{3} \pm 200$ & 0.99 \\
Internal calibration (Pss uninfected samples) & $2.00-20.00$ & $8.8 \cdot 10^{3} \pm 100$ & 0.99 \\
External calibration & $2.00-40.00$ & $1.5 \cdot 10^{4} \pm 90$ & 0.99 \\
\hline
\end{tabular}

are, likely, the dominant driving force of the binding between IAA and the polymer.

For comparison other classes of phytohormones (catechin, IBA, NAA, $\mathrm{SA}$ and $\mathrm{ABA}$ ) were used, to evaluate the polymer selectivity, finding that it can bind more than $70 \%$ of catechin, IBA, NAA, SA and ABA.

The polymer was applied to the pre-treatment of plant extracts for the identification and quantitation of IAA, and compared to traditional SPE cartridges. The LOD in real samples was $0.50 \pm 0.08 \mathrm{ng} / \mathrm{g}$; the recovery was about $50 \%$. The IAA concentration in uninfected lemon sample was $7.73 \pm 0.08 \mathrm{ng} / \mathrm{g}$, and $40.12 \pm 0.09 \mathrm{ng} / \mathrm{g}$ in Pss infected lemon sample $(\mathrm{n}=3, \alpha=0.05)$. As expected, the manipulation of IAA homeostasis of plants is, indeed, among the main strategies adopted by phytopathogenic bacteria such as Pss to increase the host susceptibility. Conversely, resistant plants are less prone to an increase of IAA following bacterial infections [3], as well as occurring in copper-treated plants for plant protection. 
Table 4

Comparison of recent methods for IAA detection in plants by HPLC coupled with UV or FD detector.

\begin{tabular}{|c|c|c|c|c|c|}
\hline Matrix & Sample pretreatment & $\begin{array}{l}\text { Analytical } \\
\text { method }\end{array}$ & Detector & $\begin{array}{l}\text { LOD } \\
(\mathrm{ng} / \mathrm{mL})\end{array}$ & Ref. \\
\hline Green seaweeds & DLLME & HPLC & UV & 1000 & {$[24]$} \\
\hline Arabidopsis thaliana & SPE (ODS-C18) & Pressurized CEC & UV & 200 & [25] \\
\hline Unicellular green algae & DLLME & HPLC & Fluorescence & 1 & [26] \\
\hline Plant xylem fluids & SPME & HPLC & UV & 0.1 & [12] \\
\hline Rose & SPE DSC-MCAX + SPE LC-NH & HPLC & $\mathrm{DAD} / \mathrm{FD}$ & 3 & [27] \\
\hline Pea, rice and wheat embryos & MIP & HPLC & UV & 3 & [17] \\
\hline Lemon & New sorbent phase & HPLC & FD & 0.5 & This work \\
\hline
\end{tabular}

In the literature many methods, characterized by complex sample treatment procedures, are reported. Thus, it is advisable a method for the rapid identification and quantification of 3-IAA in plant sample. The analytical method here proposed for the IAA determination directly on plant extracts represents a valuable, highly specific and cost-effective tool to be applied in the future to monitor the effectiveness of phytoiatric treatments alternative to copper against bacterial plant pathogens.
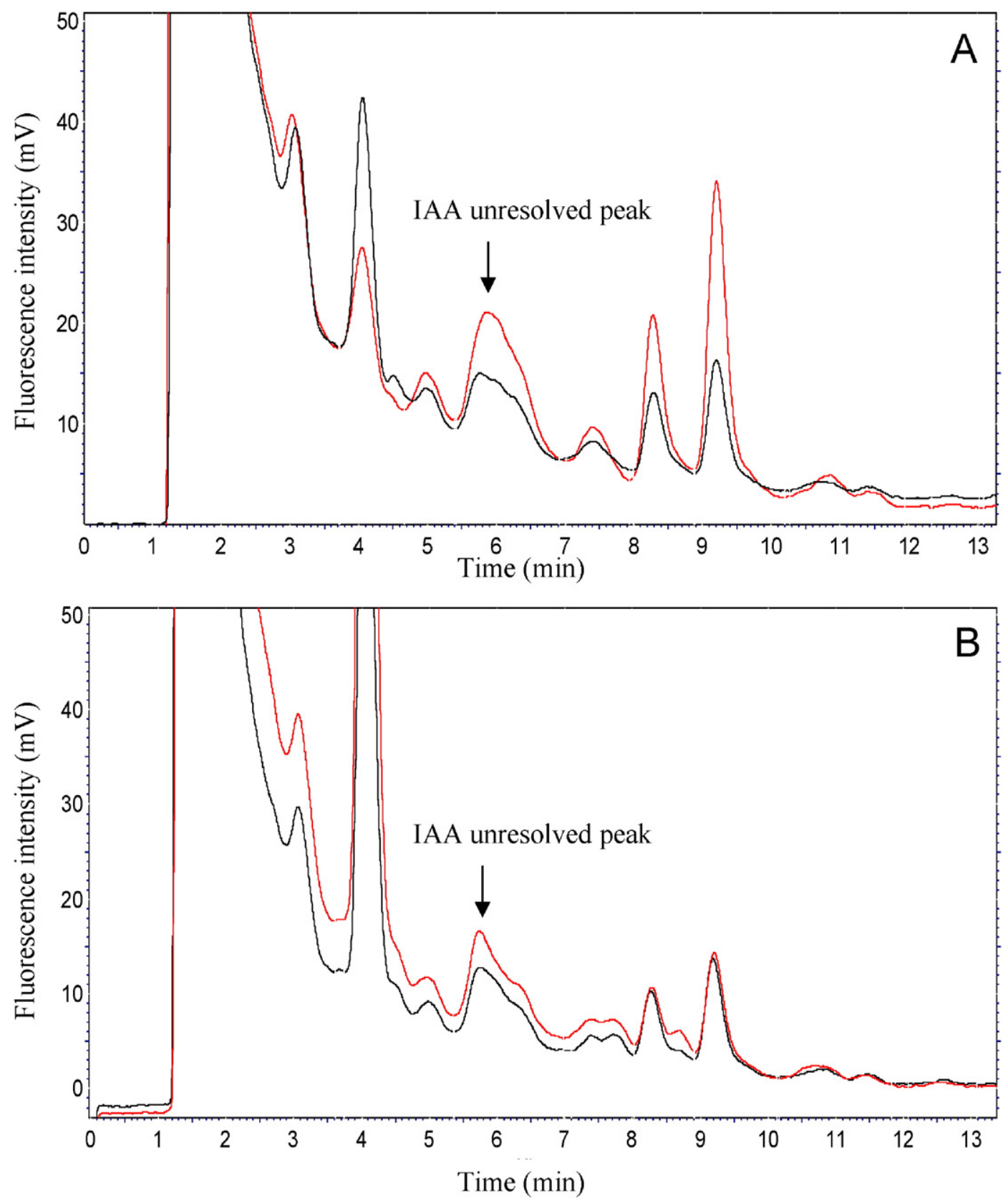

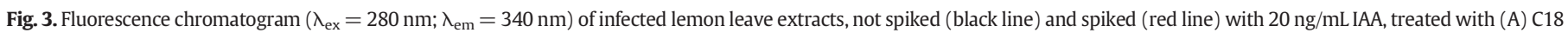
and (B) HLB cartridges. 


\section{Acknowledgments}

This work has been performed in the framework of the demonstration activities of the Life + ENV/IT/336-After Cu project. This project is aimed to the replacement of copper treatments in plants with antiinfective environmental friendly molecules against plant pathogenic bacteria.

\section{References}

[1] A. Gómez-Cadenas, J. Mehouachi, F.R. Tadeo, E. Primo-Millo, M. Talon, Hormonal regulation of fruitlet abscission induced by carbohydrate shortage in citrus, Planta 210 (2000) 636-643.

[2] S. Kessler, N. Sinha, Shaping up: the genetic control of leaf shape, Curr. Opin. Plant Biol. 7 (2004) 65-72.

[3] J. Ludwig-Müller, Bacteria and fungi controlling plant growth by manipulating auxin: balance between development and defense, J. Plant Physiol. 172 (2015) 4-12.

[4] P.J. O'Donnell, E. Schmelz, A. Block, O. Miersch, C. Wasternack, J.B. Jones, H.J. Klee, Multiple hormones act sequentially to mediate a susceptible tomato pathogen defense response, Plant Physiol. 133 (2003) 1181-1189.

[5] M. Qamar, M. Muneer, Comparative photocatalytic study of two selected pesticide derivatives, indole-3-acetic acid and indole-3-butyric acid in aqueous suspensions of titanium dioxide, J. Hazard. Mater. 120 (2005) 219-227.

[6] Z. Ma, L. Ge, A.S.Y. Lee, J.W.H. Yong, S.N. Tan, E.S. Ong, Simultaneous analysis of different classes of phytohormones in coconut (Cocos nucifera L.) water using highperformance liquid chromatography and liquid chromatography-tandem mass spectrometry after solid-phase extraction, Anal. Chim. Acta 610 (2008) 274-281.

[7] S. Liu, W. Chen, L. Qu, Y. Gai, X. Jiang, Simultaneous determination of 24 or more acidic and alkaline phytohormones in femtomole quantities of plant tissues by high-performance liquid chromatography-electrospray ionization-ion trap mass spectrometry, Anal. Bioanal. Chem. 405 (2013) 1257-1266.

[8] J. Ludwig-Müller, Auxin conjugates: their role for plant development and in the evolution of land plants, J. Exp. Bot. (2011).

[9] Y. Bai, F. Du, Y. Bai, H. Liu, Determination strategies of phytohormones: recent advances, Anal. Methods 2 (2010) 1867-1873.

[10] A. Van Eeckhaut, K. Lanckmans, S. Sarre, I. Smolders, Y. Michotte, Validation of bioanalytical LC-MS/MS assays: evaluation of matrix effects, J. Chromatogr. B 877 (2009) 2198-2207.

[11] W. Dewitte, H. Van Onckelen, Probing the distribution of plant hormones by immunocytochemistry, Plant Growth Regul. 33 (2001) 67-74.

[12] H.-T. Liu, Y.-F. Li, T.-G. Luan, C.-Y. Lan, W.-S. Shu, Simultaneous determination of phytohormones in plant extracts using SPME and HPLC, Chromatographia 66 (2007) 515-520.
[13] Y. Wu, B. Hu, Simultaneous determination of several phytohormones in natural coconut juice by hollow fiber-based liquid-liquid-liquid microextraction-high performance liquid chromatography, J. Chromatogr. A 1216 (2009) 7657-7663.

[14] N. Masque, R.M. Marce, F. Borrull, Molecularly imprinted polymers: new tailormade materials for selective solid-phase extraction, TrAC Trends Anal. Chem. 20 (2001) 477-486.

[15] I. Porobić, D. Kontrec, M. Šoškić, Molecular recognition of indole derivatives by polymers imprinted with indole-3-acetic acid: a QSPR study, Bioorg. Med. Chem. 21 (2013) 653-659.

[16] H. Yan, F. Wang, D. Han, G. Yang, Simultaneous determination of four plant hormones in bananas by molecularly imprinted solid-phase extraction coupled with high performance liquid chromatography, Analyst 137 (2012) 2884-2890.

[17] Y. Zhang, Y. Li, Y. Hu, G. Li, Y. Chen, Preparation of magnetic indole-3-acetic acid imprinted polymer beads with 4-vinylpyridine and $\beta$-cyclodextrin as binary monomer via microwave heating initiated polymerization and their application to trace analysis of auxins in plant tissues, J. Chromatogr. A 1217 (2010) 7337-7344.

[18] S.C.B. Robert, J. Umpleby, Y. Chen, R.N. Shah, K.D. Shimizu, Characterization of mo lecularly imprinted polymers with the Langmuir-Freundlich isotherm, Anal. Chem. 73 (19) (2001) 4584-4591.

[19] S. Scorrano, L. Mergola, R. Del Sole, M.R. Lazzoi, G. Vasapollo, A molecularly imprinted polymer as artificial receptor for the detection of indole-3-carbinol, J Appl. Polym. Sci. 131 (2014) (n/a-n/a).

[20] E.O. King, M.K. Ward, D.E. Raney, Two simple media for the demonstration of pyocyanin and fluorescin, J. Lab. Clin. Med. 44 (1954) 301-307.

[21] W.M. Haynes, CRC Handbook of Chemistry and Physics, CRC press, 2014.

[22] M. Yang, S. Fazio, D. Munch, P. Drumm, Impact of methanol and acetonitrile on separations based on $\pi-\pi$ interactions with a reversed-phase phenyl column, J. Chromatogr. A 1097 (2005) 124-129.

[23] P.C., B. Glick, G. Holguin, D. Penrose, Biochemical and Genetic Mechanisms Used by Plant Growth Promoting Bacteria, 199926.

[24] V. Gupta, M. Kumar, H. Brahmbhatt, C.R.K. Reddy, A. Seth, B. Jha, Simultaneous determination of different endogenetic plant growth regulators in common green seaweeds using dispersive liquid- liquid microextraction method, Plant Physiology and Biochemistry 49 (11) (2011) 1259-1263.

[25] Q. Lu, L. Zhang, L. Chen, M. Lu, P. Tong, G. Chen, Simultaneous analysis of endogenetic and ectogenic plant hormones by pressurized capillary electrochromatography, Journal of Separation Science 33 (4-5) (2010) 651-657.

[26] Q. Lu, L. Chen, M. Lu, G. Chen, L. Zhang, Extraction and Analysis of Auxins in Plants Using Dispersive Liquid-Liquid Microextraction Followed by High Performance Liquid Chromatography with Fluorescence Detection, Journal of Agricultural and Food Chemistry 58 (5) (2010) 2763-2770.

[27] R. Bosco, M. Caser, F. Vanara, V. Scariot, Development of a Rapid LC-DAD/FLD Method for the Simultaneous Determination of Auxins and Abscisic Acid in Plant Extracts, Journal of Agricultural and Food Chemistry 61 (46) (2013) 10940-10947. 